\title{
Paper
}

\section{Lighting Control Systems and End-users-Establishing an Interactive Relationship}

\author{
Amardeep M DUGAR* \\ *Lighting Research \& Design, Chennai, India
}

Received February 29, 2012, Accepted May 30, 2013

This paper is based on the authors' presentation given at the IES Annual Conference, Minneapolis MN, November, 2012.

\begin{abstract}
This paper aims to identify end-user requirements that help improve the effectiveness of lighting control systems. The crucial question posed is, what is the nature of interface designs sought by end-users for maximising interaction with lighting control systems? Literatures from the most influential studies on interactive systems are reviewed to list end-user requirements in the order of three stages of human interface with technology: Recognition, Exploration and Reliance. Results of two interactive studies with end-users are then reviewed to validate this list of requirements. The objective is to provide perspectives and themes for analysis, as well as conceptual guidance for designing lighting control interfaces that are easier to understand and use for end-users.
\end{abstract}

KEYWORDS: lighting control systems, interfaces, end-users

\section{Introduction}

Lighting controls in buildings are installed to provide end-users with "aesthetic and energy management control" over the electric lighting system ${ }^{1)}$. Moore et al. ${ }^{2)}$ argue that lighting controls provide an attractive opportunity for designers and their clients by enhancing end-user satisfaction with their local conditions, particularly when coupled with energy savings. However, studies $^{3)-6)}$ on end-users' reactions to lighting controls reveal that the complexity of contemporary control interfaces is one of the reasons for the reduced acceptability and usability of lighting control systems.

This paper explores the concept of "second-order understanding" to document end-user requirements with lighting control interfaces ${ }^{7}$. "Know thy users!" is a popular maxim in the field of interaction design. Good interface design starts with an understanding of end-users: the more one knows about them and empathises with them, the more effectively one can tailor the design for them $^{8}$. Second-order understanding listens to what endusers say they experience with lighting control interfaces. It also acknowledges their understanding as legitimate, not inferior or mistaken, even when it deviates significantly from the interface designers' intent. Interface designers could then use the documented end-user requirements in this paper for designing lighting control interfaces that are easier to understand and use than conventional interfaces.

\section{Method}

As a first step towards understanding and documenting end-user requirements, the important goals of interaction are identified so as to establish their relationship with different human skills. The important goals ascribed to while designing interfaces are "Usability and End-user Experience" ${ }^{\prime 9}$. Dugar et al..$^{5}$ associate usability with easier understanding of lighting control functions, and end-user experience with explicating the quality of end-users' experience with the lighting control interfaces such as fun of use. The different human skills to be considered while designing interfaces are cognitive skills, perceptual-motor skills and emotional skills: "in other words, knowing, doing and feeling - the wholly [sic] trinity of interaction"10). Dugar and Donn ${ }^{3)}$ have shown that cognitive and perceptual-motor skills are used for meeting usability goals to quickly and effectively learn to use the interface. Literature ${ }^{11)}$ reveals that perceptual-motor skills and emotional skills are used for meeting end-user experience goals such as pleasure of use, and enrichment of actions.

These skill-related goals are then used for listing enduser requirements based on the different stages of human interface with technology. The three different stages for human interface with technology are listed as: Recognition, Exploration, and Reliance ${ }^{7}$. Recognition refers to the stage in which end-users categorise interfaces according to what they could afford them to do, and thus require cognitive and perceptual-motor skills. Exploration follows recognition, and describes the stage 
during which end-users search for ways to handle the interface, and thus require perceptual-motor and emotional skills. Reliance is the stage in which end-users have mastered the interface and proceed naturally, seamlessly and flawlessly; however, reliance can be disrupted, causing end-users to explore alternative ways, and thus require all three skills.

Finally, this list of requirements is validated using results of two interactive studies. The first interactive

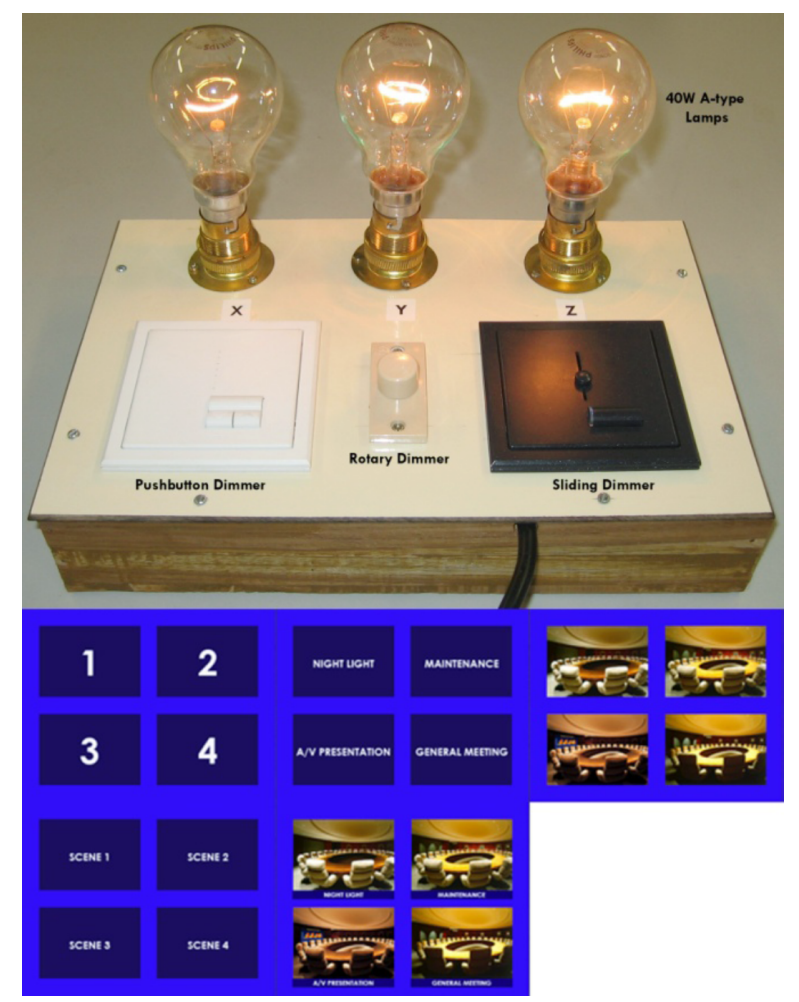

Figure 1 The manual dimming and preset control interfaces used in the first interactive study study ${ }^{45)}$ systematically engaged 30 participants from India and 30 participants from New Zealand in the lighting control process while enabling them to state their opinions about conventional interfaces, as well as needs, hopes and aspirations about their most desirable interfaces. Participants were presented with conventional interfaces for manual dimming control and the recall of preset lighting scenes. Three generic types of physical interfaces namely the pushbutton, rotary and slide were used for manually controlling luminous intensity. Five different designs of virtual interfaces were custom fabricated for recalling four preset scenes in a conference room namely Night Light, AV Presentation, Maintenance and General Meeting. The five different designs represent these scenes in the form of alphanumeric characters, typographic phrases describing the scene, iconographic images of the scene, and a combination of iconographic images and typographic phrases. The three designs of interfaces with alphanumeric characters and purely typographic phrases were derived from designs of conventional interfaces for preset control. The logic behind including these designs of conventional interfaces is to enable end-users evaluate their usability against new interface designs with iconographic images and combinations. However for statistical evaluation, only two of the three designs of conventional interfaces were used, as two designs were quite similar. Figure 1 illustrates the manual and preset control interfaces used in the first interactive study.

Tables 1 and 2 list the descriptive and inferential statistics respectively obtained from the experiment on dimming control interfaces. Participants rated the appearance of rotary and slide interfaces over the pushbutton interface as a dimming control interface. The rotary interface was rated as easier to grasp than the slide and pushbutton interfaces. The pushbutton and

Table 1 Summary of Means, Standard Deviations and Minimum-Maximum range for dimming control interfaces

\begin{tabular}{|c|c|c|c|c|c|c|c|c|c|c|}
\hline \multirow{2}{*}{ Country } & \multirow{2}{*}{ Dimension } & \multicolumn{3}{|c|}{ Pushbutton } & \multicolumn{3}{|c|}{ Rotary } & \multicolumn{3}{|c|}{ Slide } \\
\hline & & M & $\mathrm{SD}$ & Min-Max & M & $\mathrm{SD}$ & Min-Max & M & $\mathrm{SD}$ & Min-Max \\
\hline \multirow{5}{*}{ India } & Appearance & 1.500 & 0.509 & $1-2$ & 1.567 & 0.504 & $1-2$ & 1.867 & 0.346 & $1-2$ \\
\hline & Accuracy & 2.467 & 0.730 & $1-3$ & 2.067 & 0.740 & $1-3$ & 2.267 & 0.692 & $1-3$ \\
\hline & Grabbability & 1.533 & 0.507 & $1-2$ & 1.867 & 0.346 & $1-2$ & 1.433 & 0.504 & $1-2$ \\
\hline & Responsiveness & 2.333 & 0.711 & $1-3$ & 2.633 & 0.615 & $1-3$ & 2.500 & 0.509 & $2-3$ \\
\hline & Ease of use & 2.700 & 1.022 & $1-4$ & 3.667 & 0.547 & $2-4$ & 2.767 & 0.817 & $1-4$ \\
\hline \multirow{5}{*}{ New Zealand } & Appearance & 1.233 & 0.430 & $1-2$ & 1.900 & 0.305 & $1-2$ & 1.767 & 0.430 & $1-2$ \\
\hline & Accuracy & 2.533 & 0.776 & $1-3$ & 1.733 & 0.640 & $1-3$ & 2.533 & 0.629 & $1-3$ \\
\hline & Grabbability & 1.267 & 0.450 & $1-2$ & 1.967 & 0.183 & $1-2$ & 1.367 & 0.450 & $1-2$ \\
\hline & Responsiveness & 2.467 & 0.629 & $1-3$ & 2.600 & 0.675 & $1-3$ & 2.267 & 0.640 & $1-3$ \\
\hline & Ease of use & 2.400 & 0.814 & $1-4$ & 3.833 & 0.379 & $3-4$ & 2.733 & 0.640 & $2-4$ \\
\hline
\end{tabular}


Table 2 Percentages of responses and mean ranks for the different dimming control interfaces

\begin{tabular}{|c|c|c|c|c|c|c|}
\hline \multirow[b]{2}{*}{ Country } & \multirow[b]{2}{*}{ Dimension } & Pushbutton & Rotary & Slide & \multirow[b]{2}{*}{$p$} & \multirow{2}{*}{$\begin{array}{l}\text { Cochran } \\
Q(\mathrm{df}=2)\end{array}$} \\
\hline & & \multicolumn{3}{|c|}{$\begin{array}{l}\text { (Does not look like) Looks like \% } \\
\text { Or (Hard to grab) Easy to grab \% }\end{array}$} & & \\
\hline \multirow{2}{*}{ India } & Appearance & (50.0) 50.0 & (43.3) 56.7 & (13.3) 86.7 & 0.029 & 7.103 \\
\hline & Grabbability & (46.7) 53.3 & (13.3) 86.7 & (56.7) 43.3 & 0.005 & 10.692 \\
\hline \multirow{3}{*}{ New Zealand } & Appearance & (76.7) 23.3 & (10.0) 90.0 & (23.3) 76.7 & $<0.001$ & 25.846 \\
\hline & Grabbability & (73.3) 26.7 & (3.3) 96.7 & (63.3) 36.7 & $<0.001$ & 26.690 \\
\hline & & \multicolumn{3}{|l|}{ Mean ranks } & $p$ & $x^{2}(\mathrm{df}=2)$ \\
\hline \multirow{3}{*}{ India } & Accuracy & 2.27 & 1.70 & 2.03 & 0.048 & 6.083 \\
\hline & Responsiveness & 1.82 & 2.18 & 2.00 & 0.245 & 2.814 \\
\hline & Ease of use & 1.73 & 2.58 & 1.68 & $<0.001$ & 17.712 \\
\hline \multirow{3}{*}{ New Zealand } & Accuracy & 2.32 & 1.38 & 2.30 & $<0.001$ & 20.337 \\
\hline & Responsiveness & 2.00 & 2.22 & 1.78 & 0.089 & 4.829 \\
\hline & Ease of use & 1.45 & 2.82 & 1.73 & $<0.001$ & 36.725 \\
\hline
\end{tabular}

Table 3 Summary of Means, Standard Deviations and Minimum-Maximum range for preset control interfaces

\begin{tabular}{|c|c|c|c|c|c|c|}
\hline Country & Dimension & & Numbers only & Text only & Icons only & Text+icons \\
\hline \multirow{6}{*}{ India } & \multirow{3}{*}{ Accuracy } & M & 1.033 & 2.133 & 2.267 & 2.967 \\
\hline & & $\mathrm{SD}$ & 0.183 & 0.629 & 0.521 & 0.183 \\
\hline & & Min-Max & $1-2$ & $1-3$ & $1-3$ & $2-3$ \\
\hline & \multirow{3}{*}{ Learning speed } & $\mathrm{M}$ & 1.133 & 2.100 & 2.567 & 2.967 \\
\hline & & $\mathrm{SD}$ & 0.346 & 0.607 & 0.568 & 0.183 \\
\hline & & Min-Max & $1-2$ & $1-3$ & $1-3$ & $2-3$ \\
\hline \multirow{6}{*}{ New Zealand } & \multirow{3}{*}{ Accuracy } & Mean & 1.067 & 2.500 & 2.400 & 2.933 \\
\hline & & $\mathrm{SD}$ & 0.365 & 0.509 & 0.675 & 0.254 \\
\hline & & Min-Max & $1-3$ & $2-3$ & $1-3$ & $2-3$ \\
\hline & \multirow{3}{*}{ Learning speed } & Mean & 1.067 & 2.467 & 2.167 & 2.933 \\
\hline & & $\mathrm{SD}$ & 0.254 & 0.571 & 0.592 & 0.254 \\
\hline & & Min-Max & $1-2$ & $1-3$ & $1-3$ & $2-3$ \\
\hline
\end{tabular}

slide interfaces were rated as more accurate than the rotary interface for selecting their desired luminous intensity level. Participants found no difference in the responsiveness of the pushbutton, rotary and slide interfaces. The rotary interface was rated as easier to use than the slide and pushbutton interfaces. Tables 3 and 4 list the descriptive and inferential statistics respectively obtained from the experiment on preset control interfaces. The interface with a combination of iconic and textual representations of the depicted scenes was rated the most accurate, and the interface with numerical representations was rated the least accurate. Participants took the least amount of time to learn about the preset lighting scenes for recall from the interface with a combination of iconic and typographic representations of the depicted scenes, and most amount of time with the interface with only numerical representations.

The second interactive study ${ }^{6)}$ used end-user feedback from the first study to evolve a prototype design of end-users' most desirable interface called the "tangible interface." This interface consists of a central image of the lit environment with respective alphanumeric cue references of the lighting layers. "1," "2," and " 3 " are displayed on the interface to provide a direct and accurate mapping of the layers of lighting and their respective controls. This image is programmed for a synchronous gradual fade in luminous intensity and colors with the actual lighting scene when the sliders or color 
Table 4 Mean ranks for the different preset control interfaces

\begin{tabular}{|c|c|c|c|c|c|c|c|}
\hline Country & Dimensions & Numbers only & Text only & Icons only & Text+icons & \multirow{2}{*}{$p$} & \multirow{2}{*}{$x^{2}(\mathrm{df}=3)$} \\
\hline & & \multicolumn{4}{|c|}{ Mean Ranks } & & \\
\hline \multirow{2}{*}{ India } & Accuracy & 1.52 & 3.33 & 3.63 & 4.67 & $<0.001$ & 93.790 \\
\hline & Learning speed & 1.53 & 3.12 & 3.90 & 4.53 & $<0.001$ & 90.866 \\
\hline \multirow{2}{*}{ New Zealand } & Accuracy & 1.55 & 3.75 & 3.62 & 4.40 & $<0.001$ & 98.618 \\
\hline & Learning speed & 1.57 & 3.80 & 3.38 & 4.58 & $<0.001$ & 100.016 \\
\hline
\end{tabular}

Table 5 Summary of Means, Standard Deviations and Minimum-Maximum range for conventional and tangible interfaces

\begin{tabular}{|c|c|c|c|c|c|c|c|}
\hline & \multirow{2}{*}{ Dimensions } & \multicolumn{3}{|c|}{ Conventional } & \multicolumn{3}{|c|}{ Tangible } \\
\hline & & M & $\mathrm{SD}$ & Min-Max & M & $\mathrm{SD}$ & Min-Max \\
\hline \multirow{3}{*}{ Luminous intensity control } & Accuracy & 2.33 & 0.76 & $1-3$ & 2.17 & 0.77 & $1-3$ \\
\hline & Learning speed & 2.00 & 0.63 & $1-3$ & 2.86 & 0.42 & $1-3$ \\
\hline & Ease of use & 3.03 & 0.84 & $1-4$ & 2.75 & 0.87 & $1-4$ \\
\hline \multirow{3}{*}{ Luminous colour control } & Accuracy & 2.56 & 0.61 & $1-3$ & 2.81 & 0.52 & $1-3$ \\
\hline & Learning speed & 2.06 & 0.47 & $1-3$ & 3.00 & 0.00 & $3-3$ \\
\hline & Ease of use & 3.14 & 0.83 & $1-4$ & 3.56 & 0.77 & $1-4$ \\
\hline General characteristics & Responsiveness & 2.03 & 0.81 & $1-3$ & 2.64 & 0.59 & $1-3$ \\
\hline
\end{tabular}

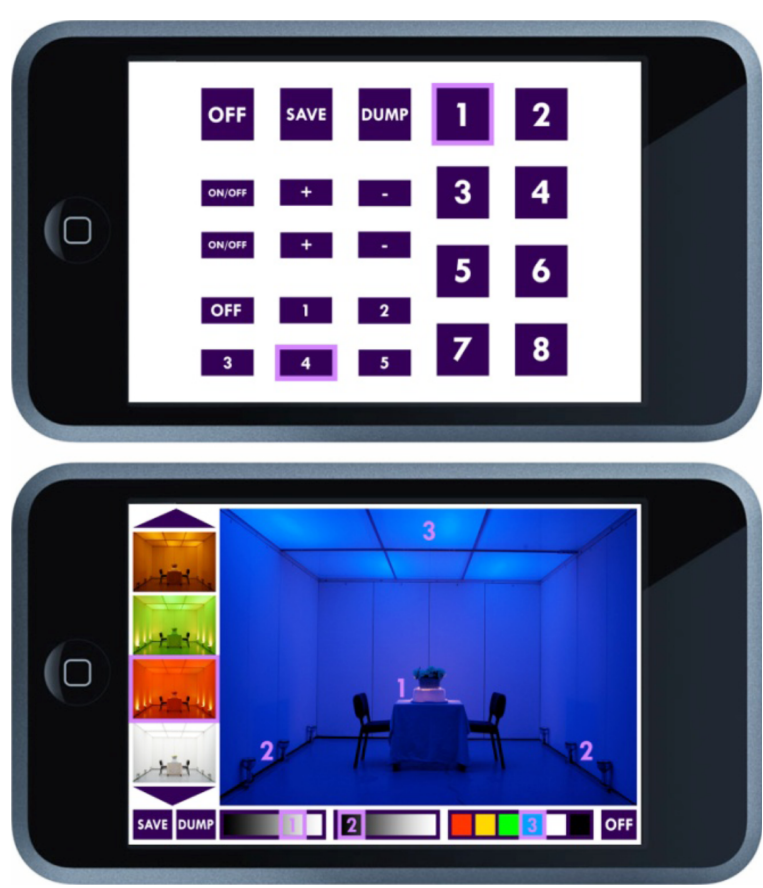

Figure 2 Prototypes of the conventional and tangible interfaces used in the second interactive study

controllers are used. Two sliders are allocated for controlling the luminous intensity of Layers -1 and -2 ; while a color palette is allocated for controlling Layer -3 : a black off button for switching off the ceiling lights and one button each for the other five colors from the test environment: amber, blue, green, red and white. Every saved scene is represented with thumbnails on a display scroll-bar, and eight scenes can be saved, scrolled and recalled using the scroll-bar. A prototype design based on designs of conventional pushbutton and touch interfaces was also developed for a comparative analysis. Both prototypes work on an iPod Touch. 36 participants from New Zealand were presented with both these prototypes for controlling the lighting of a virtual immersive environment. Figure 2 illustrates the prototypes of the conventional and tangible interfaces used in the second interactive study.

Tables 5 and 6 list the descriptive and inferential statistics of the experiment in the second interactive study respectively. Participants rated the appearance of tangible interface over the conventional interface as a device for lighting control. Participants found no difference in the accuracy of the conventional and tangible interfaces for controlling luminous intensity. However, participants commented that the accuracy of the sliders on the tangible interface could be improved with a numeric graphical scale. The tangible interface was rated more accurate than the existing interface for selecting desired luminous colours and recalling desired preset scenes. Participants were able to more accurately recall their desired preset scene in the first attempt using the tangible interface than the conventional interface. Participants took less time to learn about the luminous intensity and colour control functions from the tangible 
Table 6 Mean ranks for the conventional and tangible interfaces

\begin{tabular}{|c|c|c|c|c|c|}
\hline & \multirow{2}{*}{ Dimensions } & Conventional & Tangible & \multirow{2}{*}{$p$} & \multirow{2}{*}{$x^{2}(\mathrm{df}=1)$} \\
\hline & & \multicolumn{2}{|c|}{ Mean Ranks } & & \\
\hline \multirow{3}{*}{ Luminous intensity control } & Accuracy & 1.56 & 1.44 & 0.450 & 0.571 \\
\hline & Learning speed & 1.17 & 1.83 & $<0.001$ & 20.571 \\
\hline & Ease of use & 1.60 & 1.40 & 0.209 & 1.581 \\
\hline \multirow{3}{*}{ Luminous colour control } & Accuracy & 1.36 & 1.64 & 0.012 & 6.250 \\
\hline & Learning speed & 1.07 & 1.93 & $<0.001$ & 31.000 \\
\hline & Ease of use & 1.36 & 1.64 & 0.059 & 3.571 \\
\hline General characteristics & Responsiveness & 1.32 & 1.68 & 0.016 & 5.828 \\
\hline
\end{tabular}

interface than the conventional interface. Participants rated the tangible interface as more responsive than the conventional interface. Participants found no difference in the ease of using the conventional and tangible interfaces for controlling luminous intensity and colour. Participants preferred the overall design of the tangible interface.

\section{Requirements for recognition}

The first requirement for recognition is perceptually obvious and strong visual clues from the physical design of the lighting control interface that aid in the understanding of its function and operational mechanics. Affordance or perceived affordance refers to the perceived and actual properties of the interface, primarily those fundamental properties that determine how it could possibly be used ${ }^{12)}$. The interface should provide strong visual clues about where to grasp and how to operate it without any requirements of pictures, labels, or instructions. According to participants of the first interactive study, recognition of the dimming control function was quicker and more effective for the physical designs of the rotary and slide interfaces than the pushbutton interface. The physical designs of rotary and slide interface afforded strong visual clues as opposed to the pushbutton interface.

The second requirement for recognition is visibly clear signals via visible metaphors that provide information within a normal level understanding about the function and operation of the lighting control interface. Satisfice [a portmanteau of "satisfy" and "suffice"] is a decision-making rationalised behaviour of end-users to meet criteria for adequacy, rather than to identify an optimal solution ${ }^{13)}$. This naturalistic rational behaviour of accepting "good enough" instead of "best" is responsible for end-users' tendency of picking and trying the markedly visible control options presented by the interface first, even if it is wrong ${ }^{8}$. Poor visibility can lead to difficulty in understanding the operational use and function of interfaces ${ }^{12)}$. A semantic approach in product de- sign starts with semantics and cognition, where the product uses the knowledge and experience of the enduser to communicate information through symbols and signs ${ }^{14}$. This approach leads to the use of iconography and representation where appearance of the product and its controls become signs, often using control panels labelled with icons or may be icons themselves ${ }^{15}$.

An interface that relies on typography should be clear and provide accurate information about the available lighting control functions. The controls for different operations should be clearly labelled with texts describing what can be done, and what can be achieved for setting a particular lighting scenario. The use of graphical representations for the lighting layers along with luminous intensity and colour control could prevent end-users from selecting incorrect options, and thereby reduce chances of making mistakes. The iconography on the interface could match the learned and supposed meaning of the control function itself, apart from being recognised as a specific symbol/icon for performing that control function. According to participants of the first interactive study, recognition of their desired preset scenes was quicker and more effective for interfaces with a combination of iconographic images and typographic phrases than the alphanumeric characters. The interfaces with purely alphanumeric characters did not provide adequate information for selection.

The third requirement for recognition is a mental model that naturally maps with the lighting layers of the lit environment to mentally simulate the function and operation of the lighting control interface. Realistic representations of spatial elements readily tap into endusers' understanding of the physical world ${ }^{9}$. Conceptual modelling allows prediction of the effects of actions with the interface; conceptual models can provide end-users with a clear and unambiguous illustration of the system setup, for what each control is responsible for ${ }^{12)}$. Mapping is a technical term meaning the relationship between the controls and their movements, and the results in the world; natural mapping takes advantage of 
spatial analogies and arrangements of controls, which leads to immediate understanding of their actions in the real world ${ }^{12)}$. Bordass et al. ${ }^{16)}$ argue that mechanical controls that are direct acting, often map naturally onto the physical layout of the devices they control.

Architectural planning lends itself to natural mapping, as controllable elements like lighting have spatial meaning in the physical world ${ }^{15}$. An effective interface should have representations of lighting layers mapped in relationship to their resultant lighting control effects to enable easier recognition of the underlying lighting control concepts. Making decisions about selection and grouping of these layers, or switching and dimming them to achieve a desired luminous colour or intensity would become easier with images or icons that naturally map these functions. According to participants of the second interactive study, recognition of the dimming and present control functions was quicker and more effective from the tangible interface than the conventional interface. The realistic representations of the tangible interface were naturally mapped in relationship to their resultant lighting control effects whereas the alphanumeric representations of the conventional interface were inappropriate and incoherent.

\section{Requirements for exploration}

The first requirement for exploration are rich tactile responses to experience the physical set-up and manipulative properties of the lighting control interface. The concept of constraints refers to determining ways of restricting the kind of interaction that can take place at a given moment ${ }^{12}$. Forces and torques can be sensed to experience what type of contact it is and derive tactile information about the object's inherent properties, such as stiffness or compliance ${ }^{17}$. The interface should embody a mechanism that enables physical exploration as well as informing about the results of the process of dimming. End-users' interaction with the interface could be restricted and guided with the shape and size of its control handle along with its material properties. The shape of the control handle and its material properties would restrict and guide end-user actions respectively. The stiffness or compliance of the interfaces' control handles should enable end-users to experience their interactive and manipulative properties. Using the interface would be more satisfactory if it required a singular effortless action. According to participants of the first interactive study, exploration of the rotary and slider interfaces was more satisfactory than the pushbutton interface. The physical actions of grasping and rotating or sliding provided richer tactile responses as opposed to only pushing.

The second requirement for exploration is instantaneous visual responses to experience the conversational properties of the lighting control interface. Feedback is a well-known concept in the science of control and information theory dealing with informing end-users about what action has been done and what has been accomplished with the device, allowing them to continue with the activity ${ }^{12}$. This "success experience" provides instant gratification and confidence, which prompts endusers to keep using the device even if it gets harder later ${ }^{8)}$. End-users have a naturalistic tendency of performing an incremental style of work going back and forth to see if the result is acceptable ${ }^{8)}$. A study ${ }^{18)}$ of the "Sensoric Garden" installations that combine "multimedia with novel multi-modal interaction techniques" shows that installations providing high control and instant multi-sensorial feedback, offering simple means of interaction with a direct and transparent mapping of action and reaction were an "interactive success" that resulted in prolonged engagement by end-users.

A dynamic mapping between controls and their resultant effects in a space is an essential requirement for end-users to continue working with the interface. The interface should provide an accurate visual response every time a particular lighting level or scene has been selected, to keep end-users informed. Interacting with the interface would be fun and give confidence when it provides an instant response such as a luminous graphic that changes luminous intensity or colour every time a lighting layer is dimmed. A lack of immediate response between the mapped controls and actions performed with the interface would not help as end-users would find it difficult to remember what action has been taken. The response could be a natural consequence of end-user actions, informing them about the actions performed, and showing that the interface is responding and confirming navigation. According to participants of the second interactive study, exploration of the tangible interface was more pleasurable than the conventional interface. The tangible interface provided instantaneous visual responses every time a lighting layer or scene was selected and dimmed.

\section{Requirements for reliance}

The lone requirement for reliance is a lighting control interface that merges with the lit environment while achieving desired lighting scenarios that suit different activities. Reliance is the stage where technology disappears from our attention, where we do not need to reflect on what something means to us, and where we can address what actually matters to us $^{7}$. The most profound technologies are those that disappear by weaving themselves into the fabric of everyday life until they are indistinguishable from it ${ }^{19)}$. This change of technology's relation to end-users is described as a shift from "use" to "presence" ${ }^{20)}$. Viewing interfaces from a "use" perspective focuses on functional aspects whereas a "presence" perspective touches upon broader existen- 


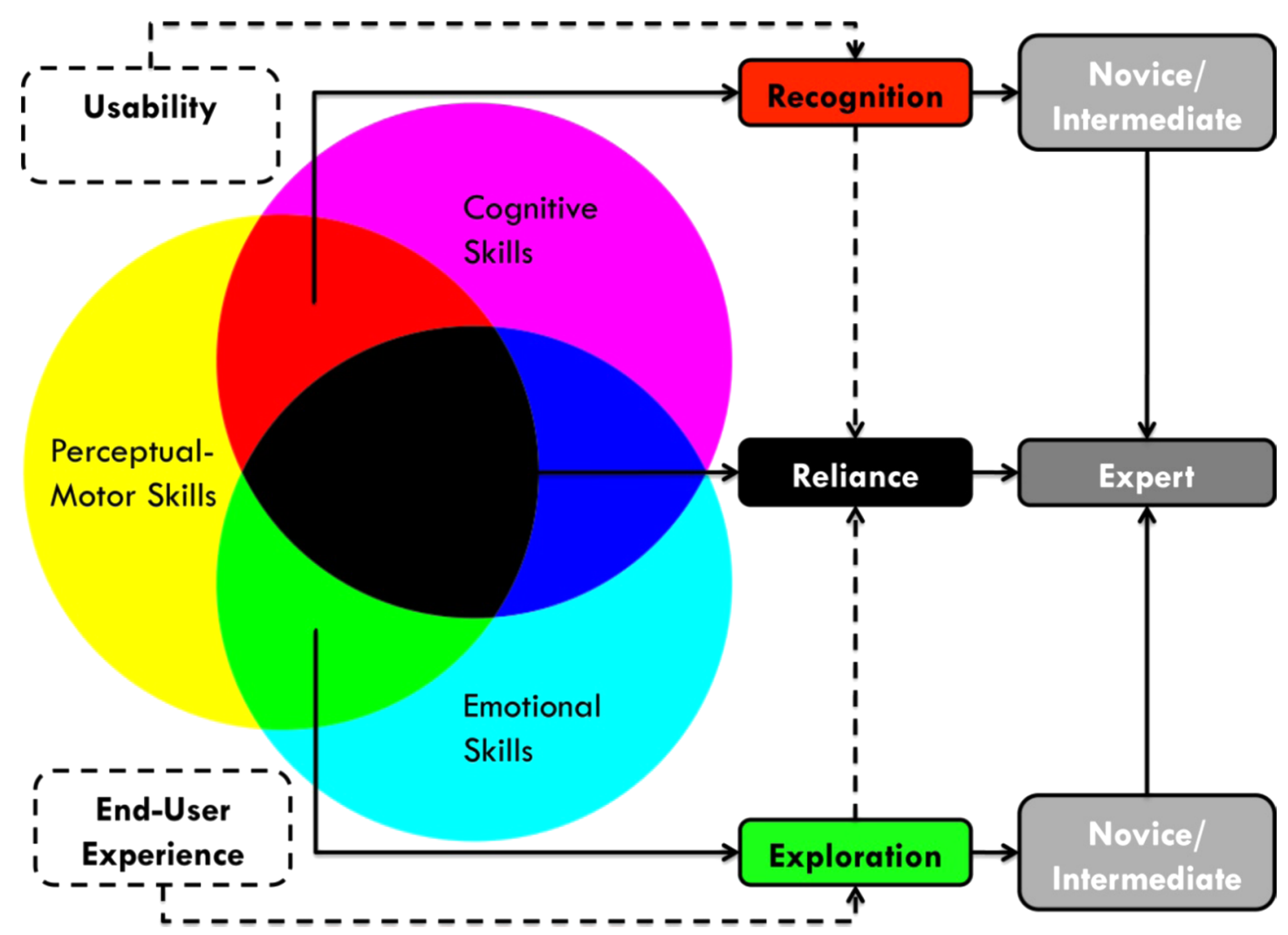

Figure 3 Relationship between the three human skills, end-user requirements and end-user attributes

tial definitions of design in the life of end-users. A popular example cited for describing technology from "use" and "presence" perspectives is the mobile phone and the communication technology it offers: its functional aspects include dialling, ringing etc., while its existential definitions include feelings of social connectivity apart from the mobile phone being a means of personal expression $^{21)}$.

The graphical representations on the interface should show the image of the space along with its lighting layers while a scene is being programmed. The representations could change from one space to another as endusers move from one space into another space. At a broader level of connectivity through the interface, endusers should be able to set a desired lighting scenario in a space from a remote location. According to participants of the second interactive study, the tangible interface was more reliant than the conventional interface. The tangible interface merged with the lit environment as the actual scenes and their realistic representations became a single entity while interacting with the lit environment.

\section{Conclusions}

On a concluding note, the more fundamental question to ask before designing a lighting control interface is how much effort are end-users willing to spend to learn the interface ${ }^{8)}$. For example, for those rare occasions when an end-user may use the interface every day for selecting different lighting scenarios for different occasions, motivation levels to learn it well may be high. On the other hand, those end-users who only use this interface sometimes will only learn it well enough to get by. And finally there are end-users who will only see the interface for a few seconds.

Therefore, it is important to identify whether most end-users can become intermediates to experts, or will remain perpetual novices. The ideal lighting control interface should be suitable to all end-user profiles ${ }^{22}$. For example, even if the novice, intermediate and expert end-users have different and conflicting requirements, the interface should ideally be designed to meet everyone's requirements. Figure 3 describes an abstract relationship between the three human skills, end-user requirements and end-user attributes.

\section{Acknowledgements}

The author thanks Michael Donn, Christopher Cuttle and Michael Dudding for advise and support during the entire research project.

\section{References}

(1) Rea, M. S. ed.: The IESNA Lighting Handbook: Reference \& Application, Illuminating Engineering So- 
ciety of North America, New York, USA (2000).

(2) Moore, T., Carter, D. J. and Slater, A. I.: A study of opinion in offices with and without user controlled lighting, Lighting Research and Technology, 36(2), pp. 131-144 (2004).

(3) Dugar, A. M. and Donn, M. R.: Tangible intervention-Improving the effectiveness of lighting control systems, Lighting Research and Technology, 43(3), pp. 381-393 (2011).

(4) Dugar, A. M.: Tangible lighting controls-a framework for improving the interactivity and usability of lighting control interfaces, Lighting-Art \& Science for International Designers, 30(2), pp. 34-42 (2010).

(5) Dugar, A. M., Donn, M. R. and Osterhaus, W: Tangible lighting controls-Reporting end-users' interactions with lighting control interfaces, LEUKOS, 8(2), pp. 123-136 (2011).

(6) Dugar, A. M., Donn, M. R. and Marshall, S.: Designing tangible lighting control interfaces, LEUKOS, 8(3), pp. 215-228 (2012).

(7) Krippendorff, K. and Butter, R.: Semantics: Meanings and contexts of artifacts, in Product Experience, Schifferstein, H. N. J. and Hekkert, P., Eds., Elsevier: San Diego, pp. 353-376 (2008).

(8) Tidwell, J.: Designing Interfaces, 1st ed, ed. Odewahn, A. and O'Brien, M., Sebastopol, CA, USA, O’Reilly Media, Inc., p. 331 (2005).

(9) Preece, J., Rogers, Y. and Sharp, H.: Interaction Design-Beyond Human-computer Interaction, 1st ed., Redvers-Mutton G., and Crockett, P., Eds., New York, USA, John Wiley \& Sons, Inc., p. 519 (2002).

(10) Overbeeke, K., et al.: Beauty in Usability, in Pleasure with Products, Beyond Usability, Green, W. and Jordan, P. Eds., Taylor \& Francis, London, UK, pp. 9-18 (2002).

(11) Djajadiningrat, T., et al.: Tangible products: redressing the balance between appearance and action, Personal Ubiquitous Computing, 8(5), pp. 294309 (2004).

(12) Norman, D. A.: The Design of Everyday Things. 1st ed., New York, USA, Doubleday/Currency, p. 257 (1988).
(13) Simon, H. A.: Models of Man: Social and Rational, New York, USA, John Wiley \& Sons (1957).

(14) Krippendorff, K. and Butter, R.: Product Semantics: Exploring the symbolic qualities of form, Innovation, The journal of the Industrial Designers Society of America, 3(2), pp. 4-9 (1984).

(15) Djajadiningrat, T., Overbeeke, K. and Wensveen, S.: But How, Donald, Tell Us How? On the Creation of Meaning in Interaction Design Through Feedforward and Inherent Feedback, in International Conference on Designing Interactive Systems, ACM Press, London, UK, pp. 285-291 (2002).

(16) Bordass, B., Leaman, A. and Bunn, R.: A Guide for Good Design and Implementation, in Controls for End Users, B.C.I. Association, Ed., BSRIA Ltd.: Berkshire, UK, p. 26 (2007).

(17) Tegin, J. and Wikander, J.: Tactile sensing in intelligent robotic manipulation - a review, Industrial Robot: An International Journal, 32(1), pp. 64-70 (2005).

(18) Hornecker, E. and Bruns, F. W.: Interactive Installations Analysis-Interaction Design of a Sensory Garden Event, in IFAC/IFIP/IFORS/IEA Symposium on the Analysis, Design and Evaluation of Human-Machine Systems, Atlanta, Georgia, USA (2004).

(19) Weiser, M.: The computer for the 21st century. SIGMOBILE Mobile Computing and Communications Review, 3(3), pp. 3-11 (1999).

(20) Hallnäs, L. and Redström, J.: From use to presence: on the expressions and aesthetics of everyday computational things. ACM Transactions on ComputerHuman Interaction, 9(2), pp. 106-124 (2002).

(21) Ross, P. and Keyson, D. V.: The case of sculpting atmospheres: towards design principles for expressive tangible interaction in control of ambient systems. Personal Ubiquitous Computing, 11(2), pp. 69-79 (2007).

(22) Thomassen, A.: In Control: Engendering a continuum of flow of a cyclic process within the context of potentially disruptive GUI interactions, in Design. Hogeschool Voor de Kunsten Utrecht, The Netherlands, p. 358 (2003). 